\title{
Fourier series of sums of products of Bernoulli and Euler/Genocchi functions
}

\author{
Taekyun Kim ${ }^{1}$, Dae San Kim ${ }^{2}$, \\ Toufik Mansour ${ }^{3}$ and Gwan-Woo Jang ${ }^{4}$ \\ ${ }^{1}$ Department of Mathematics, College of Science \\ Tianjin Polytechnic University \\ Tianjin 300160, China \\ and \\ Department of Mathematics, Kwangwoon University \\ Seoul 139-701, South Korea \\ e-mail: tkkimekw.ac.kr \\ 2 Department of Mathematics, Sogang University \\ Seoul 121-742, South Korea \\ e-mail: dskimesogang.ac.kr \\ ${ }^{3}$ Department of Mathematics, University of Haifa \\ 3498838 Haifa, Israel \\ e-mail: tmansour@univ.haifa.ac.il \\ ${ }^{4}$ Department of Mathematics, Kwangwoon University \\ Seoul 139-701, South Korea \\ e-mail: jgw5687@naver.com
}

\begin{abstract}
We study the Fourier series of functions related to sum of products of Bernoulli polynomials and either Euler or Genocchi polynomials. As consequences, several new identities for the Bernoulli, Euler, and Genocchi functions and numbers are derived.
\end{abstract}

Keywords: Fourier series, Bernoulli polynomials, Euler polynomials, Genocchi polynomials. 2010 Mathematics Subject Classification: 11B68, 42 A16. 


\section{Introduction}

We know that Bernoulli, Euler and Genocchi numbers and polynomials appear everywhere in mathematics (for example, see [1,6,14-16,18,21-23]). The Bernoulli, Euler and Genocchi numbers have been defined by the generating functions $\frac{t}{e^{t}-1}=\sum_{m \geq 0} B_{m} \frac{t^{m}}{m !}, \frac{2}{e^{t}+1}=\sum_{m \geq 0} E_{m} \frac{t^{m}}{m !}$, and $\frac{2 t}{e^{t}+1}=\sum_{m \geq 0} G_{m} \frac{t^{m}}{m !}$, respectively. The Bernoulli, Euler and Genocchi polynomials $B_{m}(x)$, $E_{m}(x)$ and $G_{m}(x)$ have been given by the generating functions [1,4-6,12-19,21-23])

$$
\frac{t}{e^{t}-1} e^{x t}=\sum_{m \geq 0} B_{m}(x) \frac{t^{m}}{m !}, \frac{2}{e^{t}+1} e^{x t}=\sum_{m \geq 0} E_{m}(x) \frac{t^{m}}{m !}, \frac{2 t}{e^{t}+1} e^{x t}=\sum_{m \geq 0} G_{m}(x) \frac{t^{m}}{m !},
$$

respectively, for any real number $x$, namely $x \in \mathbb{R}$. For instance, $B_{0}(x)=E_{0}(x)=G_{1}(x)=1$, $G_{0}(x)=0, B_{1}(x)=E_{1}(x)=x-1 / 2, B_{2}(x)=x^{2}-x+1 / 6, E_{2}(x)=x^{2}-x, G_{2}(x)=2 x-1$, $B_{3}(x)=x^{3}-3 x^{2} / 2+x / 2$, and $E_{3}(x)=x^{3}-3 x^{2} / 2+1 / 4$, and $G_{3}(x)=3 x^{2}-3 x$. Clearly, $G_{m}(x)=m E_{m-1}(x), \frac{d}{d x} G_{m}(x)=m G_{m-1}(x)$ and $G_{m}(x+1)+G_{m}(x)=2 m x^{m-1}$, for all $m \geq 1$. For $u \in \mathbb{R}$, we denote the fractional part of $u$ by $\langle u\rangle=u-\lfloor u\rfloor \in[0,1)$. In this paper, we are interested in six functions related to Bernoulli polynomials: $\tilde{\alpha}_{m}(x)=\alpha_{m}(\langle x\rangle)$, $\tilde{\beta}_{m}(x)=\beta_{m}(\langle x\rangle), \tilde{\gamma}_{m}(x)=\gamma_{m}(\langle x\rangle), \tilde{\delta}_{m}(x)=\delta_{m}(\langle x\rangle), \tilde{\eta}_{m}(x)=\eta_{m}(\langle x\rangle)$ and $\tilde{\theta}_{m}(x)=\theta_{m}(\langle x\rangle)$, where

$$
\begin{aligned}
\alpha_{m}(x) & =\sum_{k=0}^{m} B_{k}(x) E_{m-k}(x), & \beta_{m}(x) & =\sum_{k=0}^{m-1} B_{k}(x) G_{m-k}(x), \\
\gamma_{m}(x) & =\sum_{k=0}^{m} \frac{1}{k !(m-k) !} B_{k}(x) E_{m-k}(x), & \delta_{m}(x) & =\sum_{k=0}^{m-1} \frac{1}{k !(m-k) !} B_{k}(x) G_{m-k}(x), \\
\eta_{m}(x) & =\sum_{k=1}^{m-1} \frac{1}{k(m-k)} B_{k}(x) E_{m-k}(x), & \theta_{m}(x) & =\sum_{k=1}^{m-1} \frac{1}{k(m-k)} B_{k}(x) G_{m-k}(x),
\end{aligned}
$$

where $m \geq 1$, for $\alpha_{m}(x), \gamma_{m}(x)$, and $m \geq 2$, for $\beta_{m}(x), \delta_{m}(x), \eta_{m}(x), \theta_{m}(x)$. We recall the following facts about Bernoulli functions:

$$
\begin{aligned}
-m ! & \sum_{n=-\infty, n \neq 0}^{\infty} \frac{e^{2 \pi i n x}}{(2 \pi i n)^{m}}=\tilde{B}_{m}(x), m \geq 2, \\
-\sum_{n=-\infty, n \neq 0}^{\infty} \frac{e^{2 \pi i n x}}{2 \pi i n} & = \begin{cases}\tilde{B}_{1}(x), & x \notin \mathbb{Z}, \\
0, & x \in \mathbb{Z} .\end{cases}
\end{aligned}
$$

where $\tilde{B}_{m}(x)=B_{m}(\langle x\rangle)$.

The Fourier series of a periodic function $f(x)$ with period 1 is given by $\sum_{n=-\infty}^{\infty} f_{n} e^{2 \pi i n x}$, where the coefficients $f_{n}$ are given by $f_{n}=\int_{0}^{1} f(x) e^{-2 \pi i n x} d x$ (for example, see $[2,3,8-11,16$, 20, 24-26]), where $i^{2}=-1$.

The aim of this paper is to consider the Fourier series of $\tilde{\alpha}_{m}(x), \tilde{\beta}_{m}(x), \tilde{\gamma}_{m}(x), \tilde{\delta}_{m}(x), \tilde{\eta}_{m}(x)$ and $\tilde{\theta}_{m}(x)$, which lead to several new identities for the Bernoulli functions and numbers, see the next three sections. 


\section{The functions $\tilde{\alpha}_{m}$ and $\tilde{\beta}_{m}$}

In this section, we consider the functions $\tilde{\alpha}_{m},(m \geq 1)$, and $\tilde{\beta}_{m},(m \geq 2)$ on $\mathbb{R}$, each of which is periodic with period 1. The Fourier series of $\tilde{\alpha}_{m}$ and $\tilde{\beta}_{m}$ are $\sum_{n=-\infty}^{\infty} A_{n}^{(m)} e^{2 \pi i n x}$ and $\sum_{n=-\infty}^{\infty} \tilde{A}_{n}^{(m)} e^{2 \pi i n x}$, where $A_{n}^{(m)}=\int_{0}^{1} \tilde{\alpha}_{m}(x) e^{-2 \pi i n x} d x=\int_{0}^{1} \alpha_{m}(x) e^{-2 \pi i n x} d x$ and $\tilde{A}_{n}^{(m)}=$ $\int_{0}^{1} \tilde{\beta}_{m}(x) e^{-2 \pi i n x} d x=\int_{0}^{1} \beta_{m}(x) e^{-2 \pi i n x} d x$, respectively.

Define $\Lambda_{m}=2 B_{m}-E_{m-1}-2 \sum_{k=0}^{m} B_{k} E_{m-k}+2 \delta_{m, 1}$, for $m \geq 1$, and $\tilde{\Lambda}_{m}=-G_{m-1}-$ $2 \sum_{k=0}^{m-2} B_{k} G_{m-k}+2 \delta_{m, 2}$, for $m \geq 2$. To proceed further, we note the following lemma.

Lemma 1. For $m \geq 1$,

$$
\begin{aligned}
& \int_{0}^{1} \alpha_{m}(x) d x=\frac{1}{m+2} \Lambda_{m+1} \\
& \int_{0}^{1} \beta_{m}(x) d x=\frac{1}{m+2} \tilde{\Lambda}_{m+1} .
\end{aligned}
$$

Moreover, $\alpha_{m}(1)=\alpha_{m}(0)$ if and only if $\Lambda_{m}=0$, and $\beta_{m}(1)=\beta_{m}(0)$ if and only if $\tilde{\Lambda}_{m}=0$.

Proof. Recall that $\frac{d}{d x} B_{k}(x)=k B_{k-1}(x)$ and $\frac{d}{d x} E_{k}(x)=k E_{k-1}(x)$, for all $k \geq 1$. So, by the definitions, we have

$$
\begin{aligned}
\frac{d}{d x} \alpha_{m}(x) d x & =\sum_{k=0}^{m} k B_{k-1}(x) E_{m-k}(x)+\sum_{k=0}^{m}(m-k) B_{k}(x) E_{m-1-k}(x) \\
& =\sum_{k=0}^{m-1}(k+1) B_{k}(x) E_{m-1-k}(x)+\sum_{k=0}^{m-1}(m-k) B_{k}(x) E_{m-1-k}(x) \\
& =(m+1) \alpha_{m-1}(x) .
\end{aligned}
$$

Thus, $\alpha_{m}(x)=\frac{1}{m+2} \frac{d}{d x} \alpha_{m+1}(x)$, which implies

$$
\int_{0}^{1} \alpha_{m}(x) d x=\frac{1}{m+2}\left(\alpha_{m+1}(1)-\alpha_{m+1}(0)\right)=\frac{1}{m+2} \Lambda_{m+1}
$$

as claimed.

Recall that $\frac{d}{d x} G_{k}(x)=k G_{k-1}(x)$, for all $k \geq 1$. So, by the definitions,

$$
\begin{aligned}
\frac{d}{d x} \beta_{m}(x) & =\sum_{k=0}^{m} k B_{k-1}(x) G_{m-k}(x)+\sum_{k=0}^{m}(m-k) B_{k}(x) G_{m-1-k}(x) \\
& =\sum_{k=0}^{m-1}(k+1) B_{k}(x) G_{m-1-k}(x)+\sum_{k=0}^{m-1}(m-k) B_{k}(x) G_{m-1-k}(x) \\
& =(m+1) \beta_{m-1}(x) .
\end{aligned}
$$

Thus, $\beta_{m}(x)=\frac{1}{m+2} \frac{d}{d x} \beta_{m+1}(x)$, which implies

$$
\int_{0}^{1} \beta_{m}(x) d x=\frac{1}{m+2}\left(\beta_{m+1}(1)-\beta_{m+1}(0)\right)=\frac{1}{m+2} \tilde{\Lambda}_{m+1},
$$

as claimed. 
Now, we are ready to determine the Fourier coefficients $A_{n}^{(m)}$ and $\tilde{A}_{n}^{(m)}$. The case $n=0$ follows from Lemma 1, that is,

$$
A_{0}^{(m)}=\frac{1}{m+2} \Lambda_{m+1}, \quad \tilde{A}_{0}^{(m)}=\frac{1}{m+2} \tilde{\Lambda}_{m+1} .
$$

Thus, let us assume that $n \neq 0$. By Lemma 1 , we have

$$
\begin{aligned}
A_{n}^{(m)} & =\int_{0}^{1} \alpha_{m}(x) e^{-2 \pi i n x} d x=\frac{1}{2 \pi i n} \int_{0}^{1} \frac{d}{d x} \alpha_{m}(x) e^{-2 \pi i n x} d x-\left.\frac{1}{2 \pi i n} \alpha_{m}(x) e^{-2 \pi i n x}\right|_{x=0} ^{x=1} \\
& =\frac{m+1}{2 \pi i n} \int_{0}^{1} \alpha_{m-1}(x) e^{-2 \pi i n x} d x-\frac{1}{2 \pi i n}\left(\alpha_{m}(1)-\alpha_{m}(0)\right) \\
& =\frac{m+1}{2 \pi i n} A_{n}^{(m-1)}-\frac{\Lambda_{m}}{2 \pi i n} .
\end{aligned}
$$

Note that $A_{n}^{(1)}=\int_{0}^{1}(2 x-1) e^{-2 \pi i n x} d x=-\frac{2}{2 \pi i n}$ and $\tilde{A}_{n}^{(2)}=\int_{0}^{1}(3 x-3 / 2) e^{-2 \pi i n x} d x=-\frac{3}{2 \pi i n}$. So, by induction on $m$, we obtain

$$
A_{n}^{(m)}=-\frac{(m+1) !}{(2 \pi i n)^{m}}-\frac{1}{m+2} \sum_{j=1}^{m-1} \frac{(m+2)_{j}}{(2 \pi i n)^{j}} \Lambda_{m+1-j},
$$

where $m \geq 1$ and $(x)_{j}=x(x-1) \cdots(x-j+1)$ with $(x)_{0}=1$. Similarly, one can show that

$$
\tilde{A}_{n}^{(m)}=-\frac{(m+1)_{m-1}}{(2 \pi i n)^{m-1}}-\sum_{j=1}^{m-2} \frac{(m+1)_{j-1}}{(2 \pi i n)^{j}} \tilde{\Lambda}_{m+1-j}
$$

for $m \geq 2$.

Note that the functions $\tilde{\alpha}_{m}$ and $\tilde{\beta}_{m}(x)$ are piecewise $C^{\infty}$. Moreover, the functions $\tilde{\alpha}_{m}$ and $\tilde{\beta}_{m}$ are continuous for those integers $m$ with $\Lambda_{m}=0,(m \geq 1)$, and $\tilde{\Lambda}_{m}=0,(m \geq 2)$, respectively, and discontinuous with jump discontinuities at integers for those integers $m$ with $\Lambda_{m} \neq 0,(m \geq$ $1)$, and $\tilde{\Lambda}_{m} \neq 0,(m \geq 2)$, respectively.

\subsection{Case $\Lambda_{m}=0\left(\tilde{\Lambda}_{m}=0\right)$}

Assume first that $m$ is an integer with $\Lambda_{m}=0,(m \geq 1)\left(\tilde{\Lambda}_{m}=0,(m \geq 2)\right)$. Then $\alpha_{m}(1)=$ $\alpha_{m}(0)\left(\beta_{m}(1)=\beta_{m}(0)\right)$. So, the functions $\tilde{\alpha}_{m}$ and $\tilde{\beta}_{m}$ are piecewise $C^{\infty}$ and continuous. Thus, the Fourier series of $\tilde{\alpha}_{m}$ and $\tilde{\beta}_{m}$ converge uniformly to $\tilde{\alpha}_{m}$ and $\tilde{\beta}_{m}$, respectively. So, by (3), (4) and (5), we have

$$
\begin{aligned}
\tilde{\alpha}_{m}(x) & =\frac{\Lambda_{m+1}}{m+2}+\sum_{n \in \mathbb{Z}^{\prime}}\left\{-\frac{(m+1) !}{(2 \pi i n)^{m}}-\frac{1}{m+2} \sum_{j=1}^{m-1} \frac{(m+2)_{j}}{(2 \pi i n)^{j}} \Lambda_{m+1-j}\right\} e^{2 \pi i n x} \\
& =\frac{\Lambda_{m+1}}{m+2}-\frac{1}{m+2} \sum_{j=1}^{m-1}\left(\begin{array}{c}
m+2 \\
j
\end{array}\right) \Lambda_{m+1-j} j ! \sum_{n \in \mathbb{Z}^{\prime}} \frac{e^{2 \pi i n x}}{(2 \pi i n)^{j}}-(m+1) ! \sum_{n \in \mathbb{Z}^{\prime}} \frac{e^{2 \pi i n x}}{(2 \pi i n)^{m}}, \\
\tilde{\beta}_{m}(x) & =\frac{\tilde{\Lambda}_{m+1}}{m+2}+\sum_{n \in \mathbb{Z}^{\prime}}\left\{-\frac{(m+1)_{m-1}}{(2 \pi i n)^{m-1}}-\frac{1}{m+2} \sum_{j=1}^{m-2} \frac{(m+2)_{j}}{(2 \pi i n)^{j}} \tilde{\Lambda}_{m+1-j}\right\} e^{2 \pi i n x} \\
& =\frac{\tilde{\Lambda}_{m+1}}{m+2}-\frac{1}{m+2} \sum_{j=1}^{m-2}\left(\begin{array}{c}
m+2 \\
j
\end{array}\right) \tilde{\Lambda}_{m+1-j} j ! \sum_{n \in \mathbb{Z}^{\prime}} \frac{e^{2 \pi i n x}}{(2 \pi i n)^{j}}-\frac{(m+1) !}{2} \sum_{n \in \mathbb{Z}^{\prime}} \frac{e^{2 \pi i n x}}{(2 \pi i n)^{m-1}},
\end{aligned}
$$


where we define $\mathbb{Z}^{\prime}=\mathbb{Z} \backslash\{0\}$. Thus, by (1) and (2), we obtain

$$
\begin{aligned}
\tilde{\alpha}_{m}(x) & =\frac{\Lambda_{m+1}}{m+2}+\frac{1}{m+2} \sum_{j=2}^{m-1}\left(\begin{array}{c}
m+2 \\
j
\end{array}\right) \Lambda_{m+1-j} \tilde{B}_{j}(x)+(m+1) \tilde{B}_{m}(x) \\
& +\Lambda_{m} \begin{cases}\tilde{B}_{1}(x), & x \notin \mathbb{Z}, \\
0, & x \in \mathbb{Z},\end{cases} \\
\tilde{\beta}_{m}(x) & =\frac{\tilde{\Lambda}_{m+1}}{m+2}+\frac{1}{m+2} \sum_{j=2}^{m-2}\left(\begin{array}{c}
m+2 \\
j
\end{array}\right) \tilde{\Lambda}_{m+1-j} \tilde{B}_{j}(x)+\left(\begin{array}{c}
m+1 \\
2
\end{array}\right) \tilde{B}_{m-1}(x) \\
& +\tilde{\Lambda}_{m} \begin{cases}\tilde{B}_{1}(x), & x \notin \mathbb{Z}, \\
0, & x \in \mathbb{Z} .\end{cases}
\end{aligned}
$$

for all $x \in \mathbb{R}$. Thus, we can state the following results.

Theorem 2. Let $m$ be a positive integer with $\Lambda_{m}=0$. Then the function $\tilde{\alpha}_{m}(x)$ has the Fourier series expansion

$$
\tilde{\alpha}_{m}(x)=\frac{\Lambda_{m+1}}{m+2}+\sum_{n \in \mathbb{Z}^{\prime}}\left\{-\frac{(m+1) !}{(2 \pi i n)^{m}}-\frac{1}{m+2} \sum_{j=1}^{m-1} \frac{(m+2)_{j}}{(2 \pi i n)^{j}} \Lambda_{m+1-j}\right\} e^{2 \pi i n x},
$$

for all $x \in \mathbb{R}$, where the convergence is uniform. Moreover,

$$
\tilde{\alpha}_{m}(x)=\frac{\Lambda_{m+1}}{m+2}+\frac{1}{m+2} \sum_{j=2}^{m-1}\left(\begin{array}{c}
m+2 \\
j
\end{array}\right) \Lambda_{m+1-j} \tilde{B}_{j}(x)+(m+1) \tilde{B}_{m}(x) .
$$

Theorem 3. Let $m$ be an integer $\geq 2$, with $\tilde{\Lambda}_{m}=0$. Then the function $\tilde{\beta}_{m}(x)$ has the Fourier series expansion

$$
\tilde{\beta}_{m}(x)=\frac{\tilde{\Lambda}_{m+1}}{m+2}+\sum_{n \in \mathbb{Z}^{\prime}}\left\{-\frac{(m+1)_{m-1}}{(2 \pi i n)^{m-1}}-\frac{1}{m+2} \sum_{j=1}^{m-2} \frac{(m+2)_{j}}{(2 \pi i n)^{j}} \tilde{\Lambda}_{m+1-j}\right\} e^{2 \pi i n x},
$$

for all $x \in \mathbb{R}$, where the convergence is uniform. Moreover,

$$
\tilde{\beta}_{m}(x)=\frac{\tilde{\Lambda}_{m+1}}{m+2}+\frac{1}{m+2} \sum_{j=2}^{m-2}\left(\begin{array}{c}
m+2 \\
j
\end{array}\right) \tilde{\Lambda}_{m+1-j} \tilde{B}_{j}(x)+\left(\begin{array}{c}
m+1 \\
2
\end{array}\right) \tilde{B}_{m-1}(x) .
$$

\subsection{Case $\Lambda_{m} \neq 0\left(\tilde{\Lambda}_{m} \neq 0\right)$}

Assume next that $m$ is an integer with $\Lambda_{m} \neq 0,(m \geq 1)\left(\tilde{\Lambda}_{m} \neq 0,(m \geq 2)\right)$. Then $\alpha_{m}(1) \neq$ $\alpha_{m}(0)(\beta(1) \neq \beta(0))$. So, the functions $\tilde{\alpha}_{m}$ and $\tilde{\beta}_{m}$ are pointwise $C^{\infty}$ and discontinuous with jump discontinuities at integers. Thus, the Fourier series of $\tilde{\alpha}_{m}(x)$ and $\tilde{\beta}_{m}$ converge pointwise to $\tilde{\alpha}_{m}(x)$ and $\tilde{\beta}_{m}$ for all $x \notin \mathbb{Z}$, and converge to

$$
\begin{aligned}
& \frac{\alpha_{m}(1)+\alpha_{m}(0)}{2}=B_{m}-\frac{1}{2} E_{m-1}, \\
& \frac{\beta_{m}(1)+\beta_{m}(0)}{2}=B_{m-1}-\frac{1}{2} G_{m-1},
\end{aligned}
$$

for all $x \in \mathbb{Z}$. Then, by Theorems 2 and 3, we obtain the following results. 
Theorem 4. Let $m$ be a positive integer with $\Lambda_{m} \neq 0$. Then

$$
\frac{\Lambda_{m+1}}{m+2}+\sum_{n \in \mathbb{Z}^{\prime}}\left\{-\frac{(m+1) !}{(2 \pi i n)^{m}}-\frac{1}{m+2} \sum_{j=1}^{m-1} \frac{(m+2)_{j}}{(2 \pi i n)^{j}} \Lambda_{m+1-j}\right\} e^{2 \pi i n x}
$$

equals $\tilde{\alpha}_{m}(x)$ for all $x \notin \mathbb{Z}$ and $B_{m}-\frac{1}{2} E_{m-1}$ for all $x \in \mathbb{Z}$, where the convergence is pointwise. Moreover,

$$
\frac{\Lambda_{m+1}}{m+2}+\frac{1}{m+2} \sum_{j=1}^{m-1}\left(\begin{array}{c}
m+2 \\
j
\end{array}\right) \Lambda_{m+1-j} \tilde{B}_{j}(x)+(m+1) \tilde{B}_{m}(x)
$$

equals $\tilde{\alpha}_{m}(x)$ for all $x \notin \mathbb{Z}$ and

$$
\frac{\Lambda_{m+1}}{m+2}+\frac{1}{m+2} \sum_{j=2}^{m-1}\left(\begin{array}{c}
m+2 \\
j
\end{array}\right) \Lambda_{m+1-j} \tilde{B}_{j}(x)+(m+1) \tilde{B}_{m}(x)
$$

equals $B_{m}-\frac{1}{2} E_{m-1}$ for all $x \in \mathbb{Z}$.

Theorem 5. Let $m$ be an integer $\geq 2$ with $\tilde{\Lambda}_{m} \neq 0$. Then

$$
\frac{\tilde{\Lambda}_{m+1}}{m+2}+\sum_{n \in \mathbb{Z}^{\prime}}\left\{-\frac{(m+1)_{m-1}}{(2 \pi i n)^{m-1}}-\frac{1}{m+2} \sum_{j=1}^{m-1} \frac{(m+2)_{j}}{(2 \pi i n)^{j}} \tilde{\Lambda}_{m+1-j}\right\} e^{2 \pi i n x}
$$

equals $\tilde{\beta}_{m}(x)$ for all $x \notin \mathbb{Z}$ and $B_{m-1}-\frac{1}{2} G_{m-1}$ for all $x \in \mathbb{Z}$, where the convergence is pointwise. Moreover,

$$
\frac{\tilde{\Lambda}_{m+1}}{m+2}+\frac{1}{m+2} \sum_{j=1}^{m-2}\left(\begin{array}{c}
m+2 \\
j
\end{array}\right) \tilde{\Lambda}_{m+1-j} \tilde{B}_{j}(x)+\left(\begin{array}{c}
m+1 \\
2
\end{array}\right) \tilde{B}_{m-1}(x)
$$

equals $\tilde{\beta}_{m}(x)$ for all $x \notin \mathbb{Z}$ and

$$
\frac{\tilde{\Lambda}_{m+1}}{m+2}+\frac{1}{m+2} \sum_{j=2}^{m-2}\left(\begin{array}{c}
m+2 \\
j
\end{array}\right) \tilde{\Lambda}_{m+1-j} \tilde{B}_{j}(x)+\left(\begin{array}{c}
m+1 \\
2
\end{array}\right) \tilde{B}_{m-1}(x)
$$

equals $B_{m-1}-\frac{1}{2} G_{m-1}$ for all $x \in \mathbb{Z}$.

In $[13,19,20]$, it has been shown that

$$
\int_{0}^{1} \alpha_{m}(x) d x=-\frac{2 E_{m+1}}{m+1}+\frac{2}{m+1} \sum_{k=1}^{m-1} \sum_{\ell=k+1}^{m}(-1)^{k+\ell} \frac{\left(\begin{array}{c}
m+1 \\
\ell
\end{array}\right)}{\left(\begin{array}{c}
m \\
k
\end{array}\right)} B_{\ell} E_{m+1-\ell} .
$$

Thus, by Lemma 1, we establish the following identity

$$
\int_{0}^{1} \alpha_{m}(x) d x=-\frac{2 E_{m+1}}{m+1}+\frac{2}{m+1} \sum_{k=1}^{m-1} \sum_{\ell=k+1}^{m}(-1)^{k+\ell} \frac{\left(\begin{array}{c}
m+1 \\
\ell
\end{array}\right)}{\left(\begin{array}{c}
m \\
k
\end{array}\right)} B_{\ell} E_{m+1-\ell}=\frac{\Lambda_{m+1}}{m+2} .
$$

Theorems 2, 3, 4 and 5 suggest the following question: For what values of integers $m \geq 1$ does $\Lambda_{m}=0\left(\tilde{\Lambda}_{m}=0\right)$ hold? 


\section{The functions $\tilde{\gamma}_{m}$ and $\tilde{\delta}_{m}$}

In this section, we consider the functions $\tilde{\gamma}_{m},(m \geq 1)$, and $\tilde{\delta}_{m},(m \geq 2)$ on $\mathbb{R}$, each of which is periodic with period 1.

The Fourier series of $\tilde{\gamma}_{m}$ and $\tilde{\delta}_{m}$ are $\sum_{n=-\infty}^{\infty} B_{n}^{(m)} e^{2 \pi i n x}$ and $\sum_{n=-\infty}^{\infty} \tilde{B}_{n}^{(m)} e^{2 \pi i n x}$, where $B_{n}^{(m)}=$ $\int_{0}^{1} \tilde{\gamma}_{m}(x) e^{-2 \pi i n x} d x=\int_{0}^{1} \gamma_{m}(x) e^{-2 \pi i n x} d x$ and $\tilde{B}_{n}^{(m)}=\int_{0}^{1} \tilde{\delta}_{m}(x) e^{-2 \pi i n x} d x=\int_{0}^{1} \delta_{m}(x) e^{-2 \pi i n x} d x$, respectively.

Define $\Omega_{m}=\frac{2 B_{m}}{m !}-\frac{E_{m-1}}{(m-1) !}-2 \sum_{k=0}^{m} \frac{B_{k} E_{m-k}}{k !(m-k) !}+\frac{2 \delta_{m, 1}}{(m-1) !}$, for $m \geq 1$, and $\tilde{\Omega}_{m}=-2 \sum_{k=0}^{m-2} \frac{B_{k} G_{m-k}}{k !(m-k) !}-$ $\frac{G_{m-1}}{(m-1) !}+\frac{2 \delta_{m, 2}}{(m-1) !}$, for $m \geq 2$.

To proceed further, we note the following lemma.

Lemma 6. For $m \geq 1$,

$$
\begin{aligned}
& \int_{0}^{1} \gamma_{m}(x) d x=\frac{1}{2} \Omega_{m+1}, \\
& \int_{0}^{1} \delta_{m}(x) d x=\frac{1}{2} \tilde{\Omega}_{m+1},
\end{aligned}
$$

Moreover, $\gamma_{m}(1)=\gamma_{m}(0)$ if and only if $\Omega_{m}=0$, and $\delta_{m}(1)=\delta_{m}(0)$ if and only if $\tilde{\Omega}_{m}=0$.

Proof. Recall that $\frac{d}{d x} B_{k}(x)=k B_{k-1}(x)$ and $\frac{d}{d x} E_{k}(x)=k E_{k-1}(x)$, for all $k \geq 1$. So, by the definitions, we have

$$
\begin{aligned}
\frac{d}{d x} \gamma_{m}(x) & =\sum_{k=0}^{m} \frac{k B_{k-1}(x) E_{m-k}(x)}{k !(m-k) !}+\sum_{k=0}^{m} \frac{(m-k) B_{k}(x) E_{m-1-k}(x)}{k !(m-k) !} \\
& =\sum_{k=0}^{m-1} \frac{B_{k}(x) E_{m-1-k}(x)}{k !(m-1-k) !}+\sum_{k=0}^{m-1} \frac{B_{k}(x) E_{m-1-k}(x)}{k !(m-1-k) !}=2 \gamma_{m-1}(x) .
\end{aligned}
$$

Thus, $\gamma_{m}(x)=\frac{1}{2} \frac{d}{d x} \gamma_{m+1}(x)$, which gives $\int_{0}^{1} \gamma_{m}(x)=\frac{1}{2}\left(\gamma_{m+1}(1)-\gamma_{m+1}(0)\right)=\frac{1}{2} \Omega_{m+1}$, as claimed.

Recall that $\frac{d}{d x} G_{k}(x)=k G_{k-1}(x)$, for all $k \geq 1$. So, by the definitions,

$$
\begin{aligned}
\frac{d}{d x} \delta_{m}(x) & =\sum_{k=0}^{m-1} \frac{k B_{k-1}(x) G_{m-k}(x)}{k !(m-k) !}+\sum_{k=0}^{m-1} \frac{(m-k) B_{k}(x) G_{m-1-k}(x)}{k !(m-k) !} \\
& =\sum_{k=0}^{m-2} \frac{B_{k}(x) G_{m-1-k}(x)}{k !(m-1-k) !}+\sum_{k=0}^{m-2} \frac{B_{k}(x) G_{m-1-k}(x)}{k !(m-1-k) !}=2 \delta_{m-1}(x) .
\end{aligned}
$$

Thus, $\delta_{m}(x)=\frac{1}{2} \frac{d}{d x} \delta_{m+1}(x)$, which implies $\int_{0}^{1} \delta_{m}(x)=\frac{1}{2}\left(\delta_{m+1}(1)-\delta_{m+1}(0)\right)=\frac{1}{2} \tilde{\Omega}_{m+1}$, as claimed.

Now, we are ready to determine the Fourier coefficients $B_{n}^{(m)}$, and $\tilde{B}_{n}^{(m)}$. The case $n=0$ follows from Lemma 6 , that is,

$$
B_{0}^{(m)}=\frac{1}{2} \Omega_{m+1}, \quad \tilde{B}_{0}^{(m)}=\frac{1}{m+2} \tilde{\Omega}_{m+1} .
$$


Thus, let us assume that $n \neq 0$. By Lemma 6 , we have

$$
\begin{aligned}
B_{n}^{(m)} & =\int_{0}^{1} \gamma_{m}(x) e^{-2 \pi i n x} d x=\frac{1}{2 \pi i n} \int_{0}^{1} \frac{d}{d x} \gamma_{m}(x) e^{-2 \pi i n x} d x-\left.\frac{1}{2 \pi i n} \gamma_{m}(x) e^{-2 \pi i n x}\right|_{x=0} ^{x=1} \\
& =\frac{2}{2 \pi i n} \int_{0}^{1} \gamma_{m-1}(x) e^{-2 \pi i n x} d x-\frac{1}{2 \pi i n}\left(\gamma_{m}(1)-\gamma_{m}(0)\right) \\
& =\frac{2}{2 \pi i n} B_{n}^{(m-1)}-\frac{\Omega_{m}}{2 \pi i n} .
\end{aligned}
$$

Similarly,

$$
\tilde{B}_{n}^{(m)}=\frac{2}{2 \pi i n} \tilde{B}_{n}^{(m-1)}-\frac{\tilde{\Omega}_{m}}{2 \pi i n} .
$$

Note that $B_{n}^{(1)}=\int_{0}^{1}(2 x-1) e^{-2 \pi i n x} d x=-\frac{2}{2 \pi i n}$ and $\tilde{B}_{n}^{(2)}=\int_{0}^{1}(2 x-1) e^{-2 \pi i n x} d x=-\frac{2}{2 \pi i n}$. So, by induction on $m$, we obtain

$$
B_{n}^{(m)}=-\frac{1}{(\pi i n)^{m}}-\sum_{j=1}^{m-1} \frac{2^{j-1}}{(2 \pi i n)^{j}} \Omega_{m+1-j},
$$

where $m \geq 1$ and $(x)_{j}=x(x-1) \cdots(x-j+1)$ with $(x)_{0}=1$. Similarly, one can show that

$$
\tilde{B}_{n}^{(m)}=-\frac{1}{(\pi i n)^{m-1}}-\sum_{j=1}^{m-2} \frac{2^{j-1}}{(2 \pi i n)^{j}} \tilde{\Omega}_{m+1-j},
$$

for $m \geq 2$.

Note that the functions $\tilde{\gamma}_{m}$, and $\tilde{\delta}_{m}$ are piecewise $C^{\infty}$. Moreover, the functions $\tilde{\gamma}_{m}$ and $\tilde{\delta}_{m}$ are continuous for those integers $m$ with $\Omega_{m}=0,(m \geq 1)$, and $\tilde{\Omega}_{m}=0,(m \geq 2)$, respectively, and discontinuous with jump discontinuities at integers for those integers $m$ with $\Omega_{m} \neq 0,(m \geq 1)$, and $\tilde{\Omega}_{m} \neq 0,(m \geq 2)$, respectively.

\subsection{Case $\Omega_{m}=0\left(\tilde{\Omega}_{m}=0\right)$}

Assume first that $m$ is a positive integer with $\Omega_{m}=0\left(\tilde{\Omega}_{m}=0\right)$. Then $\gamma_{m}(1)=\gamma_{m}(0)\left(\delta_{m}(1)=\right.$ $\left.\delta_{m}(0)\right)$. So, the functions $\tilde{\gamma}_{m}$ and $\tilde{\delta}_{m}$ are piecewise $C^{\infty}$ and continuous. Thus, the Fourier series of $\tilde{\gamma}_{m}$ and $\tilde{\delta}_{m}$ converge uniformly to $\tilde{\gamma}_{m}$ and $\tilde{\delta}_{m}$, respectively. So, by (6), (7) and (8), we have

$$
\begin{aligned}
\tilde{\gamma}_{m}(x) & =\frac{\Omega_{m+1}}{2}-\sum_{n \in \mathbb{Z}^{\prime}}\left\{\frac{1}{(\pi i n)^{m}}+\sum_{j=1}^{m-1} \frac{2^{j-1}}{(2 \pi i n)^{j}} \Omega_{m+1-j}\right\} e^{2 \pi i n x} \\
& =\frac{\Omega_{m+1}}{2}+\sum_{j=1}^{m-1} \frac{2^{j-1}}{j !} \Omega_{m+1-j}(-j !) \sum_{n \in \mathbb{Z}^{\prime}} \frac{e^{2 \pi i n x}}{(2 \pi i n)^{j}}-2^{m} \sum_{n \in \mathbb{Z}^{\prime}} \frac{e^{2 \pi i n x}}{(2 \pi i n)^{m}} \\
\tilde{\delta}_{m}(x) & =\frac{\tilde{\Omega}_{m+1}}{2}+\sum_{n \in \mathbb{Z}^{\prime}}\left\{-\frac{2^{m-1}}{(2 \pi i n)^{m-1}}-\sum_{j=1}^{m-2} \frac{2^{j-1}}{(2 \pi i n)^{j}} \tilde{\Omega}_{m+1-j}\right\} e^{2 \pi i n x} \\
& =\frac{\tilde{\Omega}_{m+1}}{2}-\sum_{j=1}^{m-2} \frac{2^{j-1}}{j !} \tilde{\Omega}_{m+1-j} j ! \sum_{n \in \mathbb{Z}^{\prime}} \frac{e^{2 \pi i n x}}{(2 \pi i n)^{j}}-2^{m-1} \sum_{n \in \mathbb{Z}^{\prime}} \frac{e^{2 \pi i n x}}{(2 \pi i n)^{m-1}} .
\end{aligned}
$$


Thus, by (1) and (2), we obtain

$$
\begin{aligned}
& \tilde{\gamma}_{m}(x)=\frac{\Omega_{m+1}}{2}+\sum_{j=2}^{m-1} \frac{2^{j-1}}{j !} \Omega_{m+1-j} \tilde{B}_{j}(x)+\frac{2^{m}}{m !} \tilde{B}_{m}(x)+\Omega_{m} \begin{cases}\tilde{B}_{1}(x), & x \notin \mathbb{Z}, \\
0, & x \in \mathbb{Z},\end{cases} \\
& \tilde{\delta}_{m}(x)=\frac{\tilde{\Omega}_{m+1}}{2}+\sum_{j=2}^{m-2} \frac{2^{j-1}}{j !} \tilde{\Omega}_{m+1-j} \tilde{B}_{j}(x)+\frac{2^{m-1}}{(m-1) !} \tilde{B}_{m-1}(x)+\tilde{\Omega}_{m} \begin{cases}\tilde{B}_{1}(x), & x \notin \mathbb{Z}, \\
0, & x \in \mathbb{Z} .\end{cases}
\end{aligned}
$$

for all $x \in \mathbb{R}$. Thus, we can state the following results.

Theorem 7. Let $m$ be a positive integer with $\Omega_{m}=0$. Then the function $\tilde{\gamma}_{m}(x)$ has the Fourier series expansion

$$
\tilde{\gamma}_{m}(x)=\frac{\Omega_{m+1}}{2}+\sum_{n \in \mathbb{Z}^{\prime}}\left\{-\frac{1}{(\pi i n)^{m}}-\sum_{j=1}^{m-1} \frac{2^{j-1}}{(2 \pi i n)^{j}} \Omega_{m+1-j}\right\} e^{2 \pi i n x},
$$

for all $x \in \mathbb{R}$, where the convergence is uniform. Moreover,

$$
\tilde{\gamma}_{m}(x)=\sum_{j=0, j \neq 1}^{m-1} \frac{2^{j-1}}{j !} \Omega_{m+1-j} \tilde{B}_{j}(x)+\frac{2^{m}}{m !} \tilde{B}_{m}(x),
$$

for all $x \in \mathbb{R}$.

Theorem 8. Let $m$ be an integer $\geq 2$ with $\tilde{\Omega}_{m}=0$. Then the function $\tilde{\delta}_{m}(x)$ has the Fourier series expansion

$$
\tilde{\delta}_{m}(x)=\frac{\tilde{\Omega}_{m+1}}{2}+\sum_{n \in \mathbb{Z}^{\prime}}\left\{-\frac{1}{(\pi i n)^{m-1}}-\sum_{j=1}^{m-2} \frac{2^{j-1}}{(2 \pi i n)^{j}} \tilde{\Omega}_{m+1-j}\right\} e^{2 \pi i n x},
$$

for all $x \in \mathbb{R}$, where the convergence is uniform. Moreover,

$$
\tilde{\delta}_{m}(x)=\frac{\tilde{\Omega}_{m+1}}{2}+\sum_{j=2}^{m-2} \frac{2^{j-1}}{j !} \tilde{\Omega}_{m+1-j} \tilde{B}_{j}(x)+\frac{2^{m-1}}{(m-1) !} \tilde{B}_{m-1}(x),
$$

for all $x \in \mathbb{R}$.

\subsection{Case $\Omega_{m} \neq 0\left(\tilde{\Omega}_{m} \neq 0\right)$}

Assume next that $m$ is a positive integer with $\Omega_{m} \neq 0,(m \geq 1)\left(\tilde{\Omega}_{m} \neq 0,(m \geq 2)\right)$. Then $\gamma_{m}(1) \neq \gamma_{m}(0)(\delta(1) \neq \delta(0))$. So, the functions $\tilde{\gamma}_{m}$ and $\tilde{\delta}_{m}$ are piecewise $C^{\infty}$ and discontinuous with jump discontinuities at integers. Thus, the Fourier series of $\tilde{\gamma}_{m}(x)$ and $\tilde{\delta}_{m}$ converge pointwise to $\tilde{\gamma}_{m}(x)$ and $\tilde{\delta}_{m}$ for all $x \notin \mathbb{Z}$, and converge to

$$
\begin{aligned}
& \frac{\gamma_{m}(1)+\gamma_{m}(0)}{2}=\frac{B_{m}}{m !}-\frac{E_{m-1}}{2(m-1) !}=\frac{2^{m}}{m !} B_{m}, \\
& \frac{\delta_{m}(1)+\delta_{m}(0)}{2}=\frac{B_{m-1}}{(m-1) !}-\frac{G_{m-1}}{2(m-1) !}=\frac{2^{m-1}}{(m-1) !} B_{m-1},
\end{aligned}
$$

for all $x \in \mathbb{Z}$. Then, by Theorems 7 and 8 , we obtain the following results. 
Theorem 9. Let $m$ be a positive integer with $\Omega_{m} \neq 0$. Then

$$
\frac{\Omega_{m+1}}{2}-\sum_{n \in \mathbb{Z}^{\prime}}\left\{\frac{1}{(\pi i n)^{m}}+\sum_{j=1}^{m-1} \frac{2^{j-1}}{(2 \pi i n)^{j}} \Omega_{m+1-j}\right\} e^{2 \pi i n x}
$$

equals $\tilde{\gamma}_{m}(x)$ for all $x \notin \mathbb{Z}$ and $\frac{2^{m}}{m !} B_{m}$ for all $x \in \mathbb{Z}$, where the convergence is pointwise. Moreover,

$$
\sum_{j=0}^{m-1} \frac{2^{j-1}}{j !} \Omega_{m+1-j} \tilde{B}_{j}(x)+\frac{2^{m}}{m !} \tilde{B}_{m}(x)
$$

equals $\tilde{\gamma}_{m}(x)$ for all $x \notin \mathbb{Z}$ and

$$
\sum_{j=0, j \neq 1}^{m-1} \frac{2^{j-1}}{j !} \Omega_{m+1-j} \tilde{B}_{j}(x)+\frac{2^{m}}{m !} \tilde{B}_{m}(x)
$$

equals $\frac{2^{m-1}}{(m-1) !} B_{m-1}$ for all $x \in \mathbb{Z}$.

Theorem 10. Let $m$ be an integer $m \geq 2$ with $\tilde{\Omega}_{m} \neq 0$. Then

$$
\frac{\tilde{\Omega}_{m+1}}{2}-\sum_{n \in \mathbb{Z}^{\prime}}\left\{\frac{1}{(\pi i n)^{m-1}}+\sum_{j=1}^{m-2} \frac{2^{j-1}}{(2 \pi i n)^{j}} \tilde{\Omega}_{m+1-j}\right\} e^{2 \pi i n x}
$$

equals $\tilde{\delta}_{m}(x)$ for all $x \notin \mathbb{Z}$ and $\frac{2^{m-1} B_{m-1}}{(m-1) !}$ for all $x \in \mathbb{Z}$, where the convergence is pointwise. Moreover,

$$
\frac{\tilde{\Omega}_{m+1}}{2}+\sum_{j=1}^{m-2} \frac{2^{j-1}}{j !} \tilde{\Omega}_{m+1-j} \tilde{B}_{j}(x)+\frac{2^{m-1}}{(m-1) !} \tilde{B}_{m-1}(x)
$$

equals $\tilde{\delta}_{m}(x)$ for all $x \notin \mathbb{Z}$ and

$$
\frac{\tilde{\Omega}_{m+1}}{2}+\sum_{j=2}^{m-2} \frac{2^{j-1}}{j !} \tilde{\Omega}_{m+1-j} \tilde{B}_{j}(x)+\frac{2^{m-1}}{(m-1) !} \tilde{B}_{m-1}(x)
$$

equals $\frac{2^{m-1} B_{m-1}}{(m-1) !}$ for all $x \in \mathbb{Z}$.

In $[13,19,20]$, it has been shown that

$$
\int_{0}^{1} \gamma_{m}(x) d x=-\frac{2 E_{m+1}}{m+1}+\frac{2}{m+1} \sum_{k=1}^{m-1} \sum_{\ell=k+1}^{m}(-1)^{k+\ell}\left(\begin{array}{c}
m+1 \\
\ell
\end{array}\right) B_{\ell} E_{m+1-\ell} .
$$

Thus, by Lemma 6, we establish the following identity

$$
-\frac{2 E_{m+1}}{m+1}+\frac{2}{m+1} \sum_{k=1}^{m-1} \sum_{\ell=k+1}^{m}(-1)^{k+\ell}\left(\begin{array}{c}
m+1 \\
\ell
\end{array}\right) B_{\ell} E_{m+1-\ell}=\frac{\Omega_{m+1}}{2} .
$$

Theorems 7, 8, 9 and 10 suggest the following question: For what values of integers $m \geq 1$ does $\Omega_{m}=0\left(\tilde{\Omega}_{m}=0\right)$ hold? 


\section{The functions $\tilde{\eta}_{m}$ and $\tilde{\theta}_{m}$}

In this section, we consider the functions $\tilde{\eta}_{m},(m \geq 2)$ and $\tilde{\theta}_{m},(m \geq 2)$ on $\mathbb{R}$, each of which is periodic with period 1. The Fourier series of $\tilde{\eta}_{m}$ and $\tilde{\theta}_{m}$ are $\sum_{n=-\infty}^{\infty} C_{n}^{(m)} e^{2 \pi i n x}$ and $\sum_{n=-\infty}^{\infty} \tilde{C}_{n}^{(m)} e^{2 \pi i n x}$, where $C_{n}^{(m)}=\int_{0}^{1} \tilde{\eta}_{m}(x) e^{-2 \pi i n x} d x=\int_{0}^{1} \eta_{m}(x) e^{-2 \pi i n x} d x$ and $\tilde{C}_{n}^{(m)}=\int_{0}^{1} \tilde{\theta}_{m}(x) e^{-2 \pi i n x} d x=$ $\int_{0}^{1} \theta_{m}(x) e^{-2 \pi i n x} d x$, respectively.

Define $\Delta_{m}=-2 \sum_{k=1}^{m-1} \frac{B_{k} E_{m-k}}{k(m-k)}-\frac{E_{m-1}}{m-1}$ and $\tilde{\Delta}_{m}=-2 \sum_{k=1}^{m-2} \frac{B_{k} G_{m-k}}{k(m-k)}-\frac{G_{m-1}}{m-1}+\frac{2 \delta_{m, 2}}{m-1}$, for all $m \geq 2$. To proceed further, we note the following lemma.

Lemma 11. For $m \geq 2$,

$$
\begin{aligned}
& \int_{0}^{1} \eta_{m}(x) d x=\frac{\Delta_{m+1}+\frac{2}{m(m+1)} E_{m+1}}{m}, \\
& \int_{0}^{1} \theta_{m}(x) d x=\frac{\tilde{\Delta}_{m+1}+\frac{2}{m(m+1)} G_{m+1}}{m} .
\end{aligned}
$$

Moreover, $\eta_{m}(1)=\eta_{m}(0)$ if and only if $\Delta_{m}=0$, and $\theta_{m}(1)=\theta_{m}(0)$ if and only if $\tilde{\Delta}_{m}=0$.

Proof. Recall that $\frac{d}{d x} B_{k}(x)=k B_{k-1}(x)$ and $\frac{d}{d x} E_{k}(x)=k E_{k-1}(x)$, for all $k \geq 1$. So, by the definitions, we have

$$
\begin{aligned}
\frac{d}{d x} \eta_{m}(x) & =\sum_{k=1}^{m-1} \frac{k B_{k-1}(x) E_{m-k}(x)}{k(m-k)}+\sum_{k=1}^{m-1} \frac{(m-k) B_{k}(x) E_{m-1-k}(x)}{k(m-k)} \\
& =(m-1) \sum_{k=1}^{m-2} \frac{B_{k}(x) E_{m-1-k}(x)}{k(m-1-k)}+\frac{E_{m-1}(x)+B_{m-1}(x)}{m-1} \\
& =(m-1) \eta_{m-1}(x)+\frac{E_{m-1}(x)+B_{m-1}(x)}{m-1}
\end{aligned}
$$

Thus, $\eta_{m}(x)=\frac{d}{d x}\left(\eta_{m+1}(x) / m-\left(E_{m+1}(x)+B_{m+1}(x)\right) /\left(m^{2}(m+1)\right)\right)$, which gives

$$
\int_{0}^{1} \eta_{m}(x)=\frac{\Delta_{m+1}+\frac{2}{m(m+1)} E_{m+1}}{m}
$$

as claimed.

Recall that $\frac{d}{d x} G_{k}(x)=k G_{k-1}(x)$, for all $k \geq 1$. So, by the definitions,

$$
\begin{aligned}
\frac{d}{d x} \theta_{m}(x) & =\sum_{k=1}^{m-1} \frac{k B_{k-1}(x) G_{m-k}(x)}{k(m-k)}+\sum_{k=1}^{m-1} \frac{(m-k) B_{k}(x) G_{m-1-k}(x)}{k(m-k)} \\
& =(m-1) \sum_{k=1}^{m-2} \frac{B_{k}(x) G_{m-1-k}(x)}{k(m-1-k)}+\frac{G_{m-1}(x)}{m-1} \\
& =(m-1) \theta_{m-1}(x)+\frac{G_{m-1}(x)}{m-1}
\end{aligned}
$$


Thus, $\theta_{m}(x)=\frac{d}{d x}\left(\theta_{m+1}(x) / m-G_{m+1}(x) /\left(m^{2}(m+1)\right)\right)$, which implies

$$
\int_{0}^{1} \theta_{m}(x)=\frac{\tilde{\Delta}_{m+1}+\frac{2}{m(m+1)} G_{m+1}}{m},
$$

as claimed.

Now, we are ready to determine the Fourier coefficients $C_{n}^{(m)}$ and $\tilde{C}_{n}^{(m)}$. The case $n=0$ follows from Lemma 11, that is,

$$
C_{0}^{(m)}=\frac{\Delta_{m+1}+\frac{2}{m(m+1)} E_{m+1}}{m}, \quad \tilde{C}_{0}^{(m)}=\frac{\tilde{\Delta}_{m+1}+\frac{2}{m(m+1)} G_{m+1}}{m} .
$$

Thus, let us assume that $n \neq 0$. By Lemma 11, we have

$$
\begin{aligned}
C_{n}^{(m)} & =\int_{0}^{1} \eta_{m}(x) e^{-2 \pi i n x} d x=\frac{1}{2 \pi i n} \int_{0}^{1} \frac{d}{d x} \eta_{m}(x) e^{-2 \pi i n x} d x-\left.\frac{1}{2 \pi i n} \eta_{m}(x) e^{-2 \pi i n x}\right|_{x=0} ^{x=1} \\
& =\frac{m-1}{2 \pi i n} \int_{0}^{1} \eta_{m-1}(x) e^{-2 \pi i n x} d x-\frac{1}{2 \pi i n}\left(\eta_{m}(1)-\eta_{m}(0)\right) \\
& +\frac{1}{2 \pi i n(m-1)} \int_{0}^{1}\left(B_{m-1}(x)+E_{m-1}(x)\right) e^{-2 \pi i n x} d x .
\end{aligned}
$$

One shows that for $\ell \geq 1$,

$$
\begin{aligned}
& \int_{0}^{1} B_{\ell}(x) e^{-2 \pi i n x} d x= \begin{cases}-\frac{\ell !}{(2 \pi i n)^{\ell}}, & n \neq 0, \\
0, & n=0,\end{cases} \\
& \int_{0}^{1} E_{\ell}(x) e^{-2 \pi i n x} d x= \begin{cases}2 \sum_{k=1}^{\ell} \frac{(\ell)_{k-1}}{(2 \pi i n)^{k}} E_{\ell-k+1}, & n \neq 0, \\
-\frac{2}{\ell+1} E_{\ell+1}, & n=0,\end{cases} \\
& \int_{0}^{1} G_{\ell}(x) e^{-2 \pi i n x} d x= \begin{cases}2 \sum_{k=1}^{\ell-1} \frac{(\ell)_{k-1}}{(2 \pi i n)^{k}} G_{\ell-k+1}, & n \neq 0, \\
-\frac{2}{\ell+1} G_{\ell+1}, & n=0 .\end{cases}
\end{aligned}
$$

Thus,

$$
C_{n}^{(m)}=\frac{m-1}{2 \pi i n} C_{n}^{(m-1)}-\frac{\Delta_{m}}{2 \pi i n}-\frac{(m-2) !}{(2 \pi i n)^{m}}+\frac{2}{2 \pi i n(m-1)} \Phi_{m}
$$

where $\Phi_{m}=\sum_{k=1}^{m-1} \frac{(m-1)_{k-1}}{(2 \pi i n)^{k}} E_{m-k}$. Similarly,

$$
\tilde{C}_{n}^{(m)}=\frac{m-1}{2 \pi i n} \tilde{C}_{n}^{(m-1)}-\frac{\tilde{\Delta}_{m}}{2 \pi i n}+\frac{2}{2 \pi i n(m-1)} \tilde{\Phi}_{m},
$$

where $\tilde{\Omega}_{m}=\sum_{k=1}^{m-2} \frac{(m-1)_{k-1}}{(2 \pi i n)^{k}} G_{m-k}$. Note that $C_{n}^{(2)}=\int_{0}^{1}\left(x^{2}-x+1 / 4\right) e^{-2 \pi i n x} d x=-\frac{2}{(2 \pi i n)^{2}}$ and $\tilde{C}_{n}^{(2)}=\int_{0}^{1}(x-1 / 2) e^{-2 \pi i n x} d x=-\frac{1}{2 \pi i n}$. So, by induction on $m$, we obtain

$$
\begin{aligned}
C_{n}^{(m)}= & -\frac{(m-1) !}{(2 \pi i n)^{m}}-\sum_{j=1}^{m-2} \frac{(m-1)_{j-1}}{(2 \pi i n)^{j}} \Delta_{m+1-j} \\
& -\frac{(m-1) !}{(2 \pi i n)^{m}} H_{m-1}+\sum_{j=1}^{m-2} \frac{2(m-1)_{j-1}}{(2 \pi i n)^{j}(m-j)} \Phi_{m+1-j}
\end{aligned}
$$


where $m \geq 2$. Similarly, one can show that

$$
\tilde{C}_{n}^{(m)}=-\frac{(m-1) !}{(2 \pi i n)^{m-1}}-\sum_{j=1}^{m-2} \frac{(m-1)_{j-1}}{(2 \pi i n)^{j}} \tilde{\Delta}_{m+1-j}+\sum_{j=1}^{m-2} \frac{2(m-1)_{j-1}}{(2 \pi i n)^{j}(m-j)} \tilde{\Phi}_{m+1-j},
$$

for $m \geq 2$.

Lemma 12. We have

$$
\begin{aligned}
& \sum_{j=1}^{m-2} \frac{2(m-1)_{j-1}}{(2 \pi i n)^{j}(m-j)} \Phi_{m+1-j} \\
& =\frac{2}{m} \sum_{s=1}^{m-1} \frac{(m)_{s}}{(2 \pi i n)^{s}} \frac{E_{m-s+1}}{m-s+1}\left(H_{m-1}-H_{m-s}\right)-\frac{(m-1) !}{(2 \pi i n)^{m}}\left(H_{m-1}-1\right), \\
& \sum_{j=1}^{m-2} \frac{2(m-1)_{j-1}}{(2 \pi i n)^{j}(m-j)} \tilde{\Phi}_{m+1-j} \\
& =\frac{2}{m} \sum_{s=1}^{m-1} \frac{(m)_{s}}{(2 \pi i n)^{s}} \frac{G_{m-s+1}}{m-s+1}\left(H_{m-1}-H_{m-s}\right) .
\end{aligned}
$$

Proof. We present only the proof of the first identity, as that of the second one is analogous. By the definitions, we have

$$
\begin{gathered}
\sum_{j=1}^{m-2} \frac{2(m-1)_{j-1}}{(2 \pi i n)^{j}(m-j)} \Phi_{m+1-j}=\sum_{j=1}^{m-2} \frac{2(m-1)_{j-1}}{(2 \pi i n)^{j}(m-j)} \sum_{k=1}^{m-j} \frac{(m-1)_{k-1}}{(2 \pi i n)^{k}} E_{m-j-k+1} \\
=\sum_{j=1}^{m-2} \sum_{k=1}^{m-j} \frac{2(m-1)_{j+k-2}}{(2 \pi i n)^{j+k}(m-j)} E_{m-j-k+1} \\
=\frac{2}{m} \sum_{j=1}^{m-2} \sum_{k=1}^{m-j} \frac{(m)_{j+k-1}}{(2 \pi i n)^{j+k}(m-j)} E_{m-j-k+1} \\
=\frac{2}{m} \sum_{j=1}^{m-2} \sum_{s=j+1}^{m} \frac{(m)_{s-1}}{(2 \pi i n)^{s}(m-j)} E_{m-s+1} .
\end{gathered}
$$

Thus by interchanging the order of the sums, we obtain

$$
\begin{gathered}
\sum_{j=1}^{m-2} \frac{2(m-1)_{j-1}}{(2 \pi i n)^{j}(m-j)} \Phi_{m+1-j}=\frac{2}{m}\left\{\sum_{s=2}^{m-1} \frac{(m)_{s-1}}{(2 \pi i n)^{s}} E_{m-s+1} \sum_{j=1}^{s-1} \frac{1}{m-j}+\sum_{j=1}^{m-2} \frac{m !}{(2 \pi i n)^{m}(m-j)} E_{1}\right\} \\
=\frac{2}{m} \sum_{s=1}^{m-1} \frac{(m)_{s}}{(2 \pi i n)^{s}} \frac{E_{m-s+1}}{m-s+1}\left(H_{m-1}-H_{m-s}\right)-\frac{(m-1) !}{(2 \pi i n)^{m}}\left(H_{m-1}-1\right),
\end{gathered}
$$

as required. 
Hence, by (10) and (11) with using Lemma 12, we obtain

$$
\begin{aligned}
C_{n}^{(m)} & =\frac{-1}{m} \sum_{s=1}^{m-2} \frac{(m)_{s}}{(2 \pi i n)^{s}} \Delta_{m+1-s}-\frac{2(m-1) !}{(2 \pi i n)^{m}} H_{m-1} \\
& +\frac{2}{m} \sum_{s=1}^{m-2} \frac{(m)_{s}}{(2 \pi i n)^{s}} \frac{E_{m-s+1}}{m+1-s}\left(H_{m-1}-H_{m-s}\right), \\
\tilde{C}_{n}^{(m)} & =\frac{-1}{m} \sum_{s=1}^{m-2} \frac{(m)_{s}}{(2 \pi i n)^{s}} \tilde{\Delta}_{m+1-s}-\frac{(m-1) !}{(2 \pi i n)^{m-1}} H_{m-1} \\
& +\frac{2}{m} \sum_{s=1}^{m-2} \frac{(m)_{s}}{(2 \pi i n)^{s}} \frac{G_{m-s+1}}{m+1-s}\left(H_{m-1}-H_{m-s}\right),
\end{aligned}
$$

for $m \geq 2$.

Note that the functions $\tilde{\eta}_{m}$ and $\tilde{\theta}_{m}(x), m \geq 2$, are piecewise $C^{\infty}$. Moreover, the functions $\tilde{\eta}_{m}$ and $\tilde{\theta}_{m}$ are continuous for those integers $m \geq 2$ with $\Delta_{m}=0$ and $\tilde{\Delta}_{m}=0$, respectively, and discontinuous with jump discontinuities at integers for those integers $m \geq 2$ with $\Delta_{m} \neq 0$ and $\tilde{\Delta}_{m} \neq 0$, respectively.

\subsection{Case $\Delta_{m}=0\left(\tilde{\Delta}_{m}=0\right)$}

Assume first that $m$ is an integer $\geq 2$ with $\Delta_{m}=0\left(\tilde{\Delta}_{m}=0\right)$. Then $\eta_{m}(1)=\eta_{m}(0)\left(\theta_{m}(1)=\right.$ $\left.\theta_{m}(0)\right)$. So, the functions $\tilde{\eta}_{m}$ and $\tilde{\theta}_{m}$ are piecewise $C^{\infty}$ and continuous. Thus, the Fourier series of $\tilde{\eta}_{m}$ and $\tilde{\theta}_{m}$ converge uniformly to $\tilde{\eta}_{m}$ and $\tilde{\theta}_{m}$, respectively. So, by (9), (12) and (13), we have

$$
\begin{aligned}
\tilde{\eta}_{m}(x) & =\frac{\Delta_{m+1}}{m}+\frac{2 E_{m+1}}{m^{2}(m+1)} \\
& +\sum_{n \in \mathbb{Z}^{\prime}}\left\{-\frac{2(m-1) !}{(2 \pi i n)^{m}} H_{m-1}+\frac{1}{m} \sum_{s=1}^{m-2} \frac{(m)_{s}}{(2 \pi i n)^{s}}\left(2 \frac{E_{m+1-s}}{m+1-s}\left(H_{m-1}-H_{m-s}\right)-\Delta_{m+1-s}\right)\right\} e^{2 \pi i n x} \\
& =\frac{\Delta_{m+1}}{m}+\frac{2 E_{m+1}}{m^{2}(m+1)} \\
& +\frac{1}{m} \sum_{s=1}^{m-2}\left(\begin{array}{c}
m \\
s
\end{array}\right)\left(2 \frac{E_{m+1-s}}{m+1-s}\left(H_{m-1}-H_{m-s}\right)-\Delta_{m+1-s}\right) s ! \sum_{n \in \mathbb{Z}^{\prime}} \frac{e^{2 \pi i n x}}{(2 \pi i n)^{s}} \\
& -2 H_{m-1}(m-1) ! \sum_{n \in \mathbb{Z}^{\prime}} \frac{e^{2 \pi i n x}}{(2 \pi i n)^{m}}
\end{aligned}
$$

and

$$
\begin{aligned}
\tilde{\theta}_{m}(x) & =\frac{\tilde{\Delta}_{m+1}}{m}+\frac{2 G_{m+1}}{m^{2}(m+1)} \\
& +\sum_{n \in \mathbb{Z}^{\prime}}\left\{-\frac{(m-1) !}{(2 \pi i n)^{m-1}} H_{m-1}+\frac{1}{m} \sum_{s=1}^{m-2} \frac{(m)_{s}}{(2 \pi i n)^{s}}\left(2 \frac{G_{m+1-s}}{m+1-s}\left(H_{m-1}-H_{m-s}\right)-\tilde{\Delta}_{m+1-s}\right)\right\} e^{2 \pi i n x} \\
& =\frac{\tilde{\Delta}_{m+1}}{m}+\frac{2 G_{m+1}}{m^{2}(m+1)} \\
& +\frac{1}{m} \sum_{s=1}^{m-2}\left(\begin{array}{c}
m \\
s
\end{array}\right)\left(2 \frac{G_{m+1-s}}{m+1-s}\left(H_{m-1}-H_{m-s}\right)-\tilde{\Delta}_{m+1-s}\right) s ! \sum_{n \in \mathbb{Z}^{\prime}} \frac{e^{2 \pi i n x}}{(2 \pi i n)^{s}} \\
& -H_{m-1}(m-1) ! \sum_{n \in \mathbb{Z}^{\prime}} \frac{e^{2 \pi i n x}}{(2 \pi i n)^{m-1}} .
\end{aligned}
$$


Thus, by (1) and (2), we obtain

$$
\begin{aligned}
\tilde{\eta}_{m}(x) & =\frac{\Delta_{m+1}}{m}+\frac{2 E_{m+1}}{m^{2}(m+1)} \\
& -\frac{1}{m} \sum_{s=2}^{m-1}\left(\begin{array}{c}
m \\
s
\end{array}\right)\left(\frac{2 E_{m+1-s}}{m+1-s}\left(H_{m-1}-H_{m-s}\right)-\Delta_{m+1-s}\right) \tilde{B}_{s}(x)+\frac{2}{m} H_{m-1} \tilde{B}_{m}(x) \\
& +\Delta_{m} \begin{cases}\tilde{B}_{1}(x), & x \notin \mathbb{Z}, \\
0, & x \in \mathbb{Z},\end{cases} \\
\tilde{\theta}_{m}(x) & =\frac{\tilde{\Delta}_{m+1}}{m}+\frac{2 G_{m+1}}{m^{2}(m+1)} \\
& -\frac{1}{m} \sum_{s=2}^{m-1}\left(\begin{array}{l}
m \\
s
\end{array}\right)\left(\begin{array}{l}
2 G_{m+1-s} \\
m+1-s
\end{array}\left(H_{m-1}-H_{m-s}\right)-\tilde{\Delta}_{m+1-s}\right) \tilde{B}_{s}(x)+H_{m-1} \tilde{B}_{m-1}(x) \\
& +\tilde{\Delta}_{m} \begin{cases}\tilde{B}_{1}(x), & x \notin \mathbb{Z}, \\
0, & x \in \mathbb{Z},\end{cases}
\end{aligned}
$$

for all $x \in \mathbb{R}$. Thus, we can state the following results.

Theorem 13. Let $m \geq 2$ be an integer with $\Delta_{m}=0$. Then the function $\tilde{\eta}_{m}(x)$ has the Fourier series expansion

$$
\begin{aligned}
\tilde{\eta}_{m}(x) & =\frac{\Delta_{m+1}}{m}+\frac{2 E_{m+1}}{m^{2}(m+1)} \\
& +\sum_{n \in \mathbb{Z}^{\prime}}\left\{-\frac{2(m-1) !}{(2 \pi i n)^{m}} H_{m-1}+\frac{1}{m} \sum_{s=1}^{m-2} \frac{(m)_{s}}{(2 \pi i n)^{s}}\left(2 \frac{E_{m+1-s}}{m+1-s}\left(H_{m-1}-H_{m-s}\right)-\Delta_{m+1-s}\right)\right\} e^{2 \pi i n x},
\end{aligned}
$$

for all $x \in \mathbb{R}$, where the convergence is uniform. Moreover,

$$
\begin{aligned}
\tilde{\eta}_{m}(x) & =\frac{\Delta_{m+1}}{m}+\frac{2 E_{m+1}}{m^{2}(m+1)} \\
& -\frac{1}{m} \sum_{s=2}^{m-1}\left(\begin{array}{c}
m \\
s
\end{array}\right)\left(\frac{2 E_{m+1-s}}{m+1-s}\left(H_{m-1}-H_{m-s}\right)-\Delta_{m+1-s}\right) \tilde{B}_{s}(x)+\frac{2}{m} H_{m-1} \tilde{B}_{m}(x),
\end{aligned}
$$

for all $x \in \mathbb{R}$.

Theorem 14. Let $m \geq 2$ be an integer with $\tilde{\Delta}_{m}=0$. Then the function $\tilde{\theta}_{m}(x)$ has the Fourier series expansion

$$
\begin{aligned}
\tilde{\theta}_{m}(x) & =\frac{\tilde{\Delta}_{m+1}}{m}+\frac{2 G_{m+1}}{m^{2}(m+1)} \\
& +\sum_{n \in \mathbb{Z}^{\prime}}\left\{-\frac{(m-1) !}{(2 \pi i n)^{m}} H_{m-1}+\frac{1}{m} \sum_{s=1}^{m-2} \frac{(m)_{s}}{(2 \pi i n)^{s}}\left(2 \frac{G_{m+1-s}}{m+1-s}\left(H_{m-1}-H_{m-s}\right)-\tilde{\Delta}_{m+1-s}\right)\right\} e^{2 \pi i n x},
\end{aligned}
$$

for all $x \in \mathbb{R}$, where the convergence is uniform. Moreover,

$$
\begin{aligned}
\tilde{\theta}_{m}(x) & =\frac{\tilde{\Delta}_{m+1}}{m}+\frac{2 G_{m+1}}{m^{2}(m+1)} \\
& -\frac{1}{m} \sum_{s=2}^{m-2}\left(\begin{array}{c}
m \\
s
\end{array}\right)\left(\frac{2 G_{m+1-s}}{m+1-s}\left(H_{m-1}-H_{m-s}\right)-\tilde{\Delta}_{m+1-s}\right) \tilde{B}_{s}(x)+H_{m-1} \tilde{B}_{m-1}(x),
\end{aligned}
$$

for all $x \in \mathbb{R}$. 


\subsection{Case $\Delta_{m} \neq 0\left(\tilde{\Delta}_{m} \neq 0\right)$}

Assume next that $m$ is an integer $\geq 2$ with $\Delta_{m} \neq 0\left(\tilde{\Delta}_{m} \neq 0\right)$. Then $\eta_{m}(1) \neq \eta_{m}(0)(\theta(1) \neq$ $\theta(0)$ ). So, the functions $\tilde{\eta}_{m}$ and $\tilde{\theta}_{m}$ are piecewise $C^{\infty}$ and discontinuous with jump discontinuities at integers. Thus, the Fourier series of $\tilde{\eta}_{m}(x)$ and $\tilde{\theta}_{m}$ converge pointwise to $\tilde{\eta}_{m}(x)$ and $\tilde{\theta}_{m}$ for all $x \notin \mathbb{Z}$, and converge to

$$
\begin{aligned}
& \frac{\eta_{m}(1)+\eta_{m}(0)}{2}=-\frac{E_{m-1}}{2(m-1)}, \\
& \frac{\theta_{m}(1)+\theta_{m}(0)}{2}=\frac{2^{m-1}}{m-1} B_{m-1},
\end{aligned}
$$

for all $x \in \mathbb{Z}$. Then, by Theorems 13 and 14 , we obtain the following results.

Theorem 15. Let $m \geq 2$ be an integer with $\Lambda_{m} \neq 0$. Then

$$
\begin{aligned}
& \frac{\Delta_{m+1}}{m}+\frac{2 E_{m+1}}{m^{2}(m+1)} \\
& +\sum_{n \in \mathbb{Z}^{\prime}}\left\{-\frac{2(m-1) !}{(2 \pi i n)^{m}} H_{m-1}+\frac{1}{m} \sum_{s=1}^{m-2} \frac{(m)_{s}}{(2 \pi i n)^{s}}\left(2 \frac{E_{m+1-s}}{m+1-s}\left(H_{m-1}-H_{m-s}\right)-\Delta_{m+1-s}\right)\right\} e^{2 \pi i n x},
\end{aligned}
$$

equals $\tilde{\eta}_{m}(x)$ for all $x \notin \mathbb{Z}$ and $-\frac{E_{m-1}}{2(m-1)}$ for all $x \in \mathbb{Z}$, where the convergence is pointwise. Moreover,

$$
\begin{aligned}
& \frac{\Delta_{m+1}}{m}+\frac{2 E_{m+1}}{m^{2}(m+1)} \\
& -\frac{1}{m} \sum_{s=1}^{m-1}\left(\begin{array}{c}
m \\
s
\end{array}\right)\left(\frac{2 E_{m+1-s}}{m+1-s}\left(H_{m-1}-H_{m-s}\right)-\Delta_{m+1-s}\right) \tilde{B}_{s}(x)+\frac{2}{m} H_{m-1} \tilde{B}_{m}(x),
\end{aligned}
$$

equals $\tilde{\eta}_{m}(x)$ for all $x \notin \mathbb{Z}$ and

$$
\begin{aligned}
& \frac{\Delta_{m+1}}{m}+\frac{2 E_{m+1}}{m^{2}(m+1)} \\
& -\frac{1}{m} \sum_{s=2}^{m-1}\left(\begin{array}{c}
m \\
s
\end{array}\right)\left(\frac{2 E_{m+1-s}}{m+1-s}\left(H_{m-1}-H_{m-s}\right)-\Delta_{m+1-s}\right) \tilde{B}_{s}(x)+\frac{2}{m} H_{m-1} \tilde{B}_{m}(x),
\end{aligned}
$$

equals $-\frac{E_{m-1}}{2(m-1)}$ for all $x \in \mathbb{Z}$.

Theorem 16. Let $m \geq 2$ be an integer with $\tilde{\Delta}_{m} \neq 0$. Then

$$
\begin{aligned}
& \frac{\tilde{\Delta}_{m+1}}{m}+\frac{2 G_{m+1}}{m^{2}(m+1)} \\
& +\sum_{n \in \mathbb{Z}^{\prime}}\left\{-\frac{(m-1) !}{(2 \pi i n)^{m}} H_{m-1}+\frac{1}{m} \sum_{s=1}^{m-2} \frac{(m)_{s}}{(2 \pi i n)^{s}}\left(2 \frac{G_{m+1-s}}{m+1-s}\left(H_{m-1}-H_{m-s}\right)-\tilde{\Delta}_{m+1-s}\right)\right\} e^{2 \pi i n x},
\end{aligned}
$$

equals $\tilde{\theta}_{m}(x)$ for all $x \notin \mathbb{Z}$ and $\frac{2^{m-1} B_{m-1}}{m-1}$ for all $x \in \mathbb{Z}$, where the convergence is piecewise. Moreover,

$$
\begin{aligned}
& \frac{\tilde{\Delta}_{m+1}}{m}+\frac{2 G_{m+1}}{m^{2}(m+1)} \\
& -\frac{1}{m} \sum_{s=1}^{m-2}\left(\begin{array}{c}
m \\
s
\end{array}\right)\left(\frac{2 G_{m+1-s}}{m+1-s}\left(H_{m-1}-H_{m-s}\right)-\tilde{\Delta}_{m+1-s}\right) \tilde{B}_{s}(x)+H_{m-1} \tilde{B}_{m-1}(x)
\end{aligned}
$$


equals $\tilde{\theta}_{m}(x)$ for all $x \notin \mathbb{Z}$ and

$$
\frac{\tilde{\Delta}_{m+1}}{m}+\frac{2 G_{m+1}}{m^{2}(m+1)}-\frac{1}{m} \sum_{s=1}^{m-2}\left(\begin{array}{c}
m \\
s
\end{array}\right)\left(\frac{2 G_{m+1-s}}{m+1-s}\left(H_{m-1}-H_{m-s}\right)-\tilde{\Delta}_{m+1-s}\right) \tilde{B}_{s}(x)+H_{m-1} \tilde{B}_{m-1}(x)
$$

equals $\frac{2^{m-1} B_{m-1}}{m-1}$ for all $x \in \mathbb{Z}$.

In $[13,19,20]$ has been shown that

$$
\int_{0}^{1} \eta_{m}(x) d x=\frac{2}{m\left(m^{2}-1\right)} \sum_{k=0}^{m-2} \sum_{\ell=k+2}^{m}(-1)^{k+\ell+1} \frac{\left(\begin{array}{c}
m+1 \\
\ell
\end{array}\right)}{\left(\begin{array}{c}
m-2 \\
k
\end{array}\right)} B_{\ell} E_{m+1-\ell} .
$$

Thus, by Lemma 11, we establish the following identity

$$
\frac{2}{m\left(m^{2}-1\right)} \sum_{k=0}^{m-2} \sum_{\ell=k+2}^{m}(-1)^{k+\ell+1} \frac{\left(\begin{array}{c}
m+1 \\
\ell
\end{array}\right)}{\left(\begin{array}{c}
m-2 \\
k
\end{array}\right)} B_{\ell} E_{m+1-\ell}=\frac{\Delta_{m+1}}{m}+\frac{2 E_{m+1}}{m^{2}(m+1)} .
$$

Theorems 13, 14, 15 and 16 suggest the following question: For what values of integers $m \geq 2$ does $\Delta_{m}=0\left(\tilde{\Delta}_{m}=0\right)$ hold?

\section{References}

[1] Agarwal, R. P., Kim, D. S., Kim, T., \& Kwon, J. (2017) Sums of finite products of Bernoulli functions, Adv. Difference Equ., 2017, Paper No. 237, 15 pp.

[2] Apostol, T. M. (1950) Generalized Dedekind sums and transformation formulae of certain Lambert series, Duke Math. J., 17, 147-157.

[3] Bayad, A. (2011) Special values of Lerch zeta function and their Fourier expansions, Adv. Stud. Contemp. Math. (Kyungshang), 21, 1, 1-4.

[4] Carlitz, L. (1953) The reciprocity theorem for Dedekind sums, Pacific J. Math., 3, 523-527.

[5] Carlitz, L. (1968) Some unusual congruences for the Bernoulli and Genocchi numbers, Duke Math. J., 35, 563-566.

[6] Ding, D., \& Yang, J. (2010) Some identities related to the Apostol-Euler and ApostolBernoulli polynomials, Adv. Stud. Contemp. Math. (Kyungshang), 20, 1, 7-21.

[7] Dolgy, D. V., Jang, G.-W., \& Kim, T. (2017) Note on multiple $q$-zeta functions, Adv. Stud. Contemp. Math. (Kyungshang), 27, 1, 147-154.

[8] Džafarli, G. M. (1964) Fourier series for functions of the space $L_{q}$ in terms of a multiplicative system of functions, Azerbaidžan. Gos. Univ. Učen. Zap. Ser. Fiz.-Mat. i Him. Nauk, 1964, 4, 11-16 (in Russian).

[9] Gandhi, J. M. (1960) A new formula for Genocchi numbers, Math. Student, 28, 83-85. 
[10] Goes, G., \& Goes, S. (1970) Sequences of bounded variation and sequences of Fourier coefficients, I. Math. Z., 118, 93-102.

[11] Guven, A., \& Israfilov, D. M. (2009) Approximation by means of Fourier trigonometric series in weighted Orlicz spaces, Adv. Stud. Contemp. Math. (Kyungshang), 19, 2, 283-295.

[12] Jang, G.-W., Kim, D. S., Kim, T., \& Mansour, T. (2017) Fourier series of functions related to Bernoulli polynomials, Adv. Stud. Contemp. Math. (Kyungshang), 27, 1, 49-62.

[13] Kim, D. S., \& Kim, T. (2012) Bernoulli basis and the product of several Bernoulli polynomials, Int. J. Math. Sci., 2012, Art. ID 463659.

[14] Kim, T., Kim, D. S., Jang, G.-W., \& Park, J.-W. (2017) Fourier series of functions related to ordered Bell polynomials, Util. Math., 104, 67-81.

[15] Kim, T. (2010) Some identities for the Bernoulli, the Euler and the Genocchi numbers and polynomials, Adv. Stud. Contemp. Math. (Kyungshang), 20, 1, 23-28.

[16] Kim, T. (2008) Euler numbers and polynomials associated with zeta functions, Abstr. Appl. Anal., 2008, Art. ID 581582, 11 pp.

[17] Kim, T. (2008) Note on the Euler numbers and polynomials, Adv. Stud. Contemp. Math. (Kyungshang), 17, 2, 131-136.

[18] Kim, T., Choi, J., \& Kim, Y. H. (2012) A note on the values of Euler zeta functions at positive integers, Adv. Stud. Contemp. Math. (Kyungshang), 22, 1, 27-34.

[19] Kim, T., Kim, D. S., Dolgy, D. V., \& Rim, S.-H. (2013) Some identities on the Euler numbers arising from Euler basis polynomials, ARS Combinatoria, 109, 433-446.

[20] Kim, T., Kim, D. S., Rim, S.-H., \& Dolgy, D. V. (2017) Fourier series of higher-order Bernoulli functions and their applications, J. Inequal. Appl., 2017, Paper No. 8, 7 pp.

[21] Kim, T., Kim, D. S., Dolgy, D. V., \& Park, J.-W. (2017) Fourier series of sums of products of ordered Bell and poly-Bernoulli functions, J. Inequal. Appl., 2017, Paper No. 84, 17 pp.

[22] Kim, T., Kim, D. S., Jang, L. C., \& Jang, G.-W. (2017) Sums of finite products of Genocchi functions, Adv. Difference Equ., 2017, Paper No. 268, 17 pp.

[23] Shiratani, K. (1964) On some relations between Bernoulli numbers and class numbers of cyclotomic fields, Mem. Fac. Sci. Kyushu Univ. Ser. A, 18, 127-135.

[24] Watari, C. (1964) Multipliers for Walsh-Fourier series, Tôhoku Math. J., 2, 16, 239-251.

[25] Yadav, B. H. (1964) Absolute convergence of Fourier series, Thesis (Ph.D.), Maharaja Sayajirao University of Baroda, India.

[26] Zill, D. G., \& Cullen, M. R. (2006) Advanced Engineering Mathematics, Jones and Bartlett Publishers. 